\title{
WHAT PARTS OF A MEASURE'S SUPPORT ATTRACT ZEROS OF THE CORRESPONDING ORTHOGONAL POLYNOMIALS?
}

\author{
E. B. SAFF AND VILMOS TOTIK
}

(Communicated by Clifford J. Earle, Jr.)

\begin{abstract}
It is proved that each "component" of the polynomial convex hull of the support of the generating measure attracts zeros of the corresponding orthogonal polynomials. On the other hand, an example is given showing e.g. that mass points in the support need not attract zeros.
\end{abstract}

\section{Results}

Let $\mu$ be a finite Borel measure on the complex plane $\mathbb{C}$ with compact support $S(\mu):=\operatorname{supp}(\mu)$. By $\Omega=\Omega(\mu)$ we denote the outer domain of $S(\mu)$, i.e. the unbounded component of $\overline{\mathbb{C}} \backslash S(\mu)$ and by $\operatorname{Pc}(S(\mu))$ the polynomial convex hull of $S(\mu)$, i.e. $\operatorname{Pc}(S(\mu))=\overline{\mathbb{C}} \backslash \Omega$. We assume that the support of $\mu$ consists of infinitely many points. Then we can form the orthonormal polynomials

$$
p_{n}(\mu ; z)=\gamma_{n}(\mu) z^{n}+\cdots, \quad \gamma_{n}(\mu)>0
$$

with respect to $\mu$ :

$$
\int p_{n}(\mu ; z) \overline{p_{m}(\mu ; z)} d \mu(z)=\delta_{n, m},
$$

where $\delta_{n, m}=1$ if $n=m$ and $\delta_{n, m}=0$ otherwise. It easily follows from the orthogonality of these polynomials that the monic orthogonal polynomials

$$
q_{n}(\mu ; z):=\left(1 / \gamma_{n}(\mu)\right) p_{n}(\mu ; z)
$$

are the extremal polynomials for the minimization problem

$$
\min \left\{\int|P|^{2} d \mu \mid P(z)=z^{n}+\cdots\right\} .
$$

Keeping this in mind it is reasonable to expect that the zeros of $p_{n}$ are in some sense close to the support of $\mu$ so as to make the above integral as small as possible. However, the simple example of linear Lebesgue measure on the unit circle (in which case $p_{n}(\mu ; z)=z^{n}$ ) shows that the zeros can stay far away

Received by the editors July $31,1990$.

1991 Mathematics Subject Classification. Primary 42C05.

The first author's research was partially supported by NSF Grant DMS-881-4026.

The second author's research was partially supported by the Hungarian National Foundation for Research, Grant 1157. 
from the support of the generating measure. So, in what sense does the support attract zeros of the orthogonal polynomials?

It is easy to see that all zeros of $p_{n}(\mu ; z), n \in \mathbb{N}$ are contained in the convex hull of $S(\mu)$, and it is also known that for any compact set $V \subseteq \Omega$ the number of zeros of $p_{n}(\mu ; z)$ on $V$ is bounded as $n \rightarrow \infty$ (see [6] and the surveys $[2,4]$ ). Consequently, every weak* limit of the zeros (more precisely, of the normalized counting measures on the zeros) is supported on the polynomial convex hull $\operatorname{Pc}(S(\mu))$ of $S(\mu)$.

This result tells us that the polynomial convex hull of the support of $\mu$ attracts most of the zeros of $p_{n}(\mu ; \cdot)$ as $n \rightarrow \infty$. But what about parts of the support or parts of its polynomial convex hull? Our first result says that the individual "components" of the polynomial convex hull must also attract zeros.

Theorem 1. Let $\gamma$ be a simple closed Jordan curve lying in $\Omega$, and let $G$ be its interior. If $G \cap \operatorname{supp}(\mu)$ is an infinite set, then the number of zeros of $p_{n}(\mu ; \cdot)$ lying in $G$ tends to infinity with $n$.

The proof also shows that if $G \cap \operatorname{supp}(\mu)$ consists of $k<\infty$ points, then for large $n$ the orthogonal polynomial $p_{n}(\mu ; \cdot)$ has at least $k$ zeros in $G$. It may have more, as is shown by the examples of even measures on the real line, in which case the odd degree orthogonal polynomials are odd, so they have a zero at the origin regardless of whether the origin belongs to the support of the measure or not. However, we do not know any example in which a point outside the polynomial convex hull attracts zeros of $p_{n}(\mu ; \cdot)$ for all large $n$ (and not just for odd $n$ 's, as in the above example).

Let us immediately mention that the above theorem cannot be extended to ensuring any density of the zeros inside $\gamma$. In fact, the construction of [3, Theorem 2.1.4] can be easily modified to yield the following: if $S$ is any polynomially convex compact subset of the plane, then there is a (discrete) measure $\mu$ on $S$ such that if $\nu$ is any probability measure with support in $S$, then for some infinite sequence $N$ the zeros of the orthogonal polynomials $\left\{p_{n}(\mu ; \cdot)\right\}_{n \in N}$ have asymptotic distribution equal to $\nu$.

We have mentioned that the monic orthogonal polynomials minimize the integral in (1), but this does not necessarily mean that parts of the support attract zeros (only parts of its polynomial convex hull do so, according to Theorem 1). Now what about large concentration of masses in the generating measure? For example, does a point mass in $\mu$ necessarily attract at least one zero of the orthogonal polynomials? Our second theorem shows that in general nothing similar to that can be claimed.

Theorem 2. Let $\rho$ be the measure on the unit circle given by the density

$$
\frac{1}{2 \pi} \sin ^{2} \frac{\theta}{2} \text {. }
$$

If $\sigma$ is any measure with compact support in the open unit disk, then there is a $\lambda>0$ such that with $\mu=\rho+\lambda \sigma$ all the zeros of the orthogonal polynomials $p_{n}(\mu ; \cdot)$ approach the unit circle as $n \rightarrow \infty$.

The last statement means that if $R<1$, then for large $n$ the polynomial $p_{n}(\mu ; \cdot)$ has no zero in $\Delta_{R}:=\{z|| z \mid \leq R\}$. Note, however, that all the zeros of $p_{n}(\mu ; \cdot)$ must lie in the unit disk. It is also easy to prove that the zeros 
of $p_{n}(\mu ; \cdot)$ are asymptotically uniformly distributed (with regard to their argument).

If in Theorem 2 we choose $\sigma$ to be a discrete measure, then we get a $\mu$ for which mass points do not attract zeros of the corresponding orthogonal polynomials. If we choose $\sigma$ to have infinite support, then Theorem 2 also shows that the assumption $\gamma \subseteq \Omega$ is essential in Theorem 1; it cannot be replaced by $\gamma \subseteq \mathbb{C} \backslash \operatorname{supp}(\mu)$.

\section{Proof of Theorem 1}

Let us assume for an indirect proof that for some subsequence $N$ of the natural numbers each of the polynomials $p_{n}(\mu ; \cdot), n \in N$ has exactly $k<\infty$ zeros in $G$. We can assume that these zeros converge to some points $X_{1}, \ldots, X_{k} \in$ $G \cup \gamma$ as $n \rightarrow \infty, n \in N$. Let $\nu_{\left.p_{n}(\mu ; \cdot)\right)}$ be the measure that places mass $1 / n$ to each zero of $p_{n}(\mu ; \cdot)$ counting multiplicity (this is the so-called normalized counting measure on the zeros), and let $\gamma_{n}(\mu)$ be the leading coefficient of $p_{n}(\mu ; \cdot)$. By replacing $N$ by a suitable subsequence, we can assume without loss of generality that the sequences $\left\{\nu_{p_{n}(\mu ; \cdot)}\right\}_{n \in N}$ and $\left\{\gamma_{n}(\mu)^{1 / n}\right\}_{n \in N}$ converge:

$$
\nu_{p_{n}(\mu ; \cdot)} \rightarrow \nu, \quad \gamma_{n}(\mu)^{1 / n} \rightarrow e^{c}, \quad \text { as } n \rightarrow \infty, n \in N,
$$

where $\nu$ is a probability measure with support in $\operatorname{Pc}(S(\mu)), c \in \mathbb{R} \cup\{\infty\}$, and the first convergence is understood in the weak* topology on measures. We remark, that here $c$ cannot be $-\infty$ (see e.g. [4, Corollary 2.4] or [3, Corollary 1.1.7]).

Suppose first that $c<\infty$. For the logarithmic potential

$$
p(\nu ; z):=\int \log \frac{1}{|z-t|} d \nu(t)
$$

of $\nu$, we know that

$$
c-p(\nu ; z) \geq 0
$$

for $z \in \bar{\Omega}$ (see [3, (1.3.30) and Corollary 1.1.5]). By our assumption $\operatorname{supp}(\nu) \cap$ $G=\varnothing$, and so the left-hand side in (2) is harmonic in $G$. Furthermore

$$
\lim _{n \rightarrow \infty, n \in N}\left|p_{n}(\mu ; z)\right|^{1 / n}=\exp (c-p(\nu ; z))
$$

uniformly on compact subsets of $G \backslash\left\{X_{1}, \ldots, X_{k}\right\}$. The relations (2) and (3) show that we must have

$$
c-p(\nu ; z)=0 \quad \text { for } z \in(\partial \bar{\Omega} \cap G) \backslash\left\{X_{1}, \ldots, X_{k}\right\},
$$

otherwise the orthonormal polynomials $p_{n}(\mu ; \cdot)$ would have $L^{2}(\mu)$ norm greater than 1 for large $n \in N$. By continuity this equality holds true for all $z \in \partial \bar{\Omega} \cap G$ except perhaps for those isolated points of $\operatorname{Pc}(S(\mu))$ that are among the $X_{i}$ 's.

Now we distinguish two cases.

Case 1. $G \cap \operatorname{Int}(\operatorname{Pc}(S(\mu)))=\varnothing$, where Int means the two-dimensional interior of the set in question. Then (2) is true for all $z \in G$, so from (4) and the minimum principle for harmonic functions we can conclude

$$
c-p(\nu ; z)=0
$$


in $G$. But here the left-hand side is harmonic in $\Omega$, so from (5), (2), and $G \cap \Omega \neq \varnothing$, we can derive (5) for all $z \in \Omega$ which is clearly impossible.

Case 2. $G \cap \operatorname{Int}(\operatorname{Pc}(S(\mu)))=\varnothing$. On the boundary of the set $G \cap \operatorname{Int}(\operatorname{Pc}(S(\mu)))$ we have (5) (see (4)) from which we can deduce (2) for all $z \in \operatorname{Pc}(S(\mu)) \cap G$ via the maximum principle (see also (2)). Hence, (2) holds for all $z \in G$ and the rest of the argument is the same as before.

Finally, if $c=\infty$, then $\left|p_{n}(\mu ; z)\right|, n \in N$ is exponentially large in a neighborhood of some $z_{0} \in S(\mu) \cap G \backslash\left\{X_{1}, \ldots, X_{k}\right\}$ which is not possible, so this case cannot occur.

The obtained contradictions prove Theorem 1 .

\section{Proof of Theorem 2}

Without loss of generality we can assume that the measure $\sigma$ has total mass at most 1 . For clearer notation let us denote $p_{n}(\rho ; z)$ by $\phi_{n}(z)$. These orthogonal polynomials have been mentioned by $P$. Turán [5] and one can easily check from the relations

$$
\int z^{l} \overline{z^{k}} d \rho(z)= \begin{cases}0 & \text { if }|l-k|>1 \\ 1 / 2 & \text { if }|l-k|=0 \\ -1 / 4 & \text { if }|l-k|=1\end{cases}
$$

that

$$
\begin{aligned}
\phi_{n}(z) & =2 \frac{1+2 z+\cdots+(n+1) z^{n}}{\sqrt{(n+1)(n+2)}}=2 \frac{(n+1) z^{n+2}-(n+2) z^{n+1}+1}{\sqrt{(n+1)(n+2)}(1-z)^{2}} \\
& =\frac{2}{\sqrt{(n+1)(n+2)}(1-z)^{2}}+r_{n}(z)
\end{aligned}
$$

where

$$
\left|r_{n}(z)\right| \leq 5|z|^{n+1} /|1-z|^{2} \quad \text { for }|z| \leq 1 .
$$

We also need the reciprocal polynomials

$$
\phi_{n}^{*}(z):=z^{n} \phi_{n}\left(\frac{1}{z}\right)=2 \frac{(n+1)-(n+2) z+z^{n+2}}{\sqrt{(n+1)(n+2)}(1-z)^{2}}=\frac{2}{1-z}+\tilde{r}_{n}(z),
$$

where

$$
\left|\tilde{r}_{n}(z)\right| \leq \frac{10}{(n+1)|1-z|^{2}} \quad \text { for }|z| \leq 1 .
$$

If we write the monic polynomials

$$
q_{n}(z):=q_{n}(\mu ; z)=\left(1 / \gamma_{n}(\mu)\right) p_{n}(\mu ; z)
$$

in the form

$$
q_{n}(z)=a_{n} \phi_{n}(z)+a_{n-1} \phi_{n-1}(z)+\cdots+a_{0} \phi_{0}(z),
$$

then $a_{n}=1 / \gamma_{n}(\rho)=\frac{1}{2} \sqrt{(n+2) /(n+1)}$, and from the orthogonality of $q_{n}$ to each $\phi_{k}, k<n$, in $L^{2}(\mu)$ we get

$$
a_{k}=-\lambda \int q_{n} \overline{\phi_{k}} d \sigma \text {. }
$$


Hence

$$
q_{n}(z)=\frac{1}{\gamma_{n}(\rho)} \phi_{n}(z)-\lambda \int q_{n}(t)\left(\sum_{k=0}^{n-1} \overline{\phi_{k}(t)} \phi_{k}(z)\right) d \sigma(t) .
$$

Here the reproducing kernel

$$
s_{n-1}(t, z):=\sum_{k=0}^{n-1} \overline{\phi_{k}(t)} \phi_{k}(z)
$$

has the representation (see [1, Lemma 1.10])

$$
s_{n-1}(t, z)=\frac{\overline{\phi_{n}^{*}(t)} \phi_{n}^{*}(z)-\overline{\phi_{n}(t)} \phi_{n}(z)}{1-\bar{t} z} .
$$

Now it easily follows from our estimates on $\phi_{n}$ and on $\phi_{n}^{*}$, that the sequence $\left\{s_{n-1}(t, z)\right\}_{n=1}^{\infty}$ is bounded if $t$ and $z$ are restricted to a compact subset of the open unit disk. Furthermore, if $R_{0}<1$ is fixed then there exists a constant $K_{0}$ (say, $5 /\left(1-R_{0}\right)^{2}$ ) with the following property: for every $R<1$ there exists a number $n_{R}$ such that independently of $|t| \leq R_{0}$ and $|z| \leq R$, the inequality

$$
\left|s_{n-1}(t, z)\right| \leq K_{0} n\left|\phi_{n}(z)\right|
$$

holds for all $n \geq n_{R}$.

Let us now choose $R_{0}$ so that the support of the measure $\sigma$ is contained in the disk $\Delta_{R_{0}}:=\left\{z|| z \mid \leq R_{0}\right\}$. Then $\left|s_{n-1}(t, z)\right| \leq K_{1}$ for $t, z \in \Delta_{R_{0}}$ with some constant $K_{1}$. Hence it follows from the representation (6) that

$$
\max _{z \in \Delta_{R_{0}}}\left|q_{n}(z)\right| \leq \max _{z \in \Delta_{R_{0}}}\left|\phi_{n}(z)\right| / \gamma_{n}(\rho)+\lambda K_{1} \max _{z \in \Delta_{R_{0}}}\left|q_{n}(z)\right|,
$$

and so, if $\lambda<1 / 2 K_{1}$, then

$$
\max _{z \in \Delta_{R_{0}}} n\left|q_{n}(z)\right| \leq 2 \max _{z \in \Delta_{R_{0}}} n\left|\phi_{n}(z)\right| / \gamma_{n}(\rho) \leq K_{2},
$$

follows, where the constant $K_{2}$ depends only on $R_{0}$.

Now let $R<1$ be arbitrary. Using (7), the preceding inequality, and once again the representation in (6), we get for $z \in \Delta_{R}$ and $n \geq n_{R}$ that

$$
\left|n q_{n}(z)-n \phi_{n}(z) / \gamma_{n}(\rho)\right| \leq \lambda K_{0} K_{2} n\left|\phi_{n}(z)\right| \leq 2 \lambda K_{0} K_{2} n\left|\phi_{n}(z)\right| / \gamma_{n}(\rho),
$$

from which we can see that if $\lambda<1 / 4 K_{0} K_{2}$ and $\phi_{n}(z)$ has no zeros in $\Delta_{R}$, then the same is true of $q_{n}(z)$. But it is immediate from the explicit representation of $\phi_{n}$ that it will not have zeros in $\Delta_{R}$ for large $n$ and this completes the proof.

\section{REFERENCES}

1. G. Freud, Orthogonal polynomials, Akad. Kiadó, Budapest, 1971.

2. E. B. Saff, Orthogonal polynomials from a complex perspective, Orthogonal Polynomials: Theory and Practice (P. Nevai, ed.), Kluwer Academic, Dordrecht, 1990, pp. 363-393.

3. H. Stahl and V. Totik, General orthogonal polynomials, Encyclopedia of Mathematics, Cambridge Univ. Press, New York (to appear).

4. _,$N$ th root asymptotic behavior of orthonormal polynomials, Orthogonal Polynomials: Theory and Practice (P. Nevai, ed.), Kluwer Academic, Dordrecht, 1990, pp. 395-417.

5. P. Turán, Some open problems of approximation theory, Mat. Lapok 25 (1974), 21-75. (Hungarian)

6. H. Widom, Polynomials associated with measures in the complex plane, J. Math. Mech. 16 (1967), 997-1013. 
Department of Mathematics, University of South Florida, Tampa, Florida 33620

Bolyai Institute, Szeged, Aradi V. tere 1, 6720, Hungary

AND

Department of Mathematics, University of South Florida, Tampa, Florida 33620 